\title{
CONVERGENCE RESULTS FOR COMPOUND POISSON DISTRIBUTIONS AND APPLICATIONS TO THE STANDARD LURIA-DELBRÜCK DISTRIBUTION
}

\author{
M. MÖHLE, ${ }^{*}$ Eberhard Karls Universität Tübingen
}

\begin{abstract}
We provide a scaling for compound Poisson distributions that leads (under certain conditions on the Fourier transform) to a weak convergence result as the parameter of the distribution tends to infinity. We show that the limiting probability measure belongs to the class of stable Cauchy laws with Fourier transform $t \mapsto \exp (-c|t|-\mathrm{i} a t \log |t|)$. We apply this convergence result to the standard discrete Luria-Delbrück distribution and derive an integral representation for the corresponding limiting density, as an alternative to that found in a closely related paper of Kepler and Oprea. Moreover, we verify local convergence and we derive an integral representation for the distribution function of the limiting continuous Luria-Delbrück distribution.
\end{abstract}

Keywords: Compound Poisson distribution; continuous Luria-Delbrück distribution; Fourier transform; stable distribution; weak convergence

2000 Mathematics Subject Classification: Primary 60F05; 92D15

Secondary 60E10; 92D10

\section{Introduction}

One way to define the standard Luria-Delbrück (LD) distribution with parameter $m>0$ is via its generating function

$$
\sum_{n=0}^{\infty} p_{n} s^{n}:=(1-s)^{m(1-s) / s} .
$$

The probabilities $p_{n}=p_{n}(m)$ depend on $m$. Models in population biology that lead to the standard LD distribution are described in [6] and [9], for example. The name of this distribution goes back to the original work of Salvadore Luria and Max Delbrück [11]. The LD distribution is of interest in biological applications, as it provides a basis for procedures that estimate mutation rates [1], [2]. Unfortunately, the probabilities $p_{n}, n \in \mathbb{N}_{0}:=\{0,1,2, \ldots\}$, of the LD distribution are not simple to compute, in particular for large parameter $m$. Ma et al. [12] found the recursion

$$
p_{0}:=\mathrm{e}^{-m}, \quad p_{n}=\frac{m}{n} \sum_{i=1}^{n} \frac{p_{n-i}}{i+1} \quad \text { for } n \in \mathbb{N}:=\{1,2, \ldots\} .
$$

Based on this recursion, properties of the LD distribution can be derived. For example, it was shown that, for fixed parameter $m$, the asymptotics $\lim _{n \rightarrow \infty} n(n+1) p_{n}(m)=m$ holds. For more details of the tail behavior we refer the reader to [6] and [14].

Received 4 April 2003; revision received 23 December 2004.

* Postal address: Mathematisches Institut, Eberhard Karls Universität Tübingen, Auf der Morgenstelle 10, 72076

Tübingen, Germany. Email address: martin.moehle@uni-tuebingen.de 
In contrast to the situation in which $m$ is fixed and $n$ is large, we are interested in the asymptotic behavior of the LD distribution for large parameter $m$. An important question is whether the LD distribution, properly scaled, converges to some limiting distribution as the parameter $m$ tends to infinity. The papers of Pakes [13] and Angerer [2] show that such a scaling exists. For another paper on matters closely related to this question, see [7].

As the LD distribution is a special compound Poisson distribution, it is natural to ask whether such scalings and convergence results can be extended to more general classes of compound Poisson distribution. In Section 2, such convergence results are presented. The main convergence theorem (Theorem 2.2) provides the appropriate scaling and characterizes the limiting distribution via its Fourier transform. In Sections 3 and 4, these convergence results are applied to the LD distribution and further properties of the LD distribution are derived. The paper concludes with a discussion in Section 5.

\section{Convergence of compound Poisson distributions}

Compound-Poisson-distributed random variables are (by definition) of the form

$$
Y_{m}:=\sum_{k=1}^{K} X_{k},
$$

where $\left(X_{k}\right)_{k \in \mathbb{N}}$ is a sequence of independent, identically distributed random variables and $K$ is a Poisson random variable, with parameter $m>0$, independent of the sequence $\left(X_{k}\right)_{k \in \mathbb{N}}$. The Fourier transform $\varphi_{m}: \mathbb{R} \rightarrow \mathbb{C}$ of $Y_{m}$ is

$$
\varphi_{m}(t):=\mathrm{E}\left(\mathrm{e}^{\mathrm{i} t Y_{m}}\right)=\exp (m(\varphi(t)-1))
$$

where $\varphi$ denotes the Fourier transform of $X:=X_{1}$. Compound Poisson distributions appear in many fields, for example those of compound Poisson approximations and large deviation principles, and have been the subject of intense research. In this paper, we are simply interested in the behavior of $Y_{m}$ for large parameter $m$. The following convergence theorem (Theorem 2.1) is the analog of the classical law of large numbers and the classical central limit theorem. It states, in particular, that $Y_{m}$ is asymptotically normal provided that $X$ has a finite and nonvanishing second moment. Although the result is well known from the literature, we present a simple proof based on Fourier analysis, as this technique will be used again later in the paper.

Theorem 2.1. (a) If $\mathrm{E}(|X|)<\infty$ then $Y_{m} / m$ converges in probability to $\mathrm{E}(X)$.

(b) If $0<\mathrm{E}\left(X^{2}\right)<\infty$ then $Y_{m}$ is asymptotically normal, i.e.

$$
\frac{Y_{m}-m \mathrm{E}(X)}{\sqrt{m \mathrm{E}\left(X^{2}\right)}}
$$

converges in distribution to the standard normal distribution.

Remark 2.1. Owing to the fact that $Y_{m}$ is a random sum of independent, identically distributed variables, the scaling variance $\operatorname{var}\left(Y_{m}\right)=m \mathrm{E}\left(X^{2}\right)$ in part (b) of the theorem is larger than the value $m \operatorname{var}(X)$, which one would expect from the classical central limit theorem for a deterministic sum of independent, identically distributed variables. 
Proof of Theorem 2.1. (a) Fix $t \in \mathbb{R}$ and consider the Fourier transform of $Y_{m} / m$. Obviously, $\mathrm{E}\left(\mathrm{e}^{\mathrm{i} t Y_{m} / m}\right)=\mathrm{e}^{m(\varphi(x)-1)}$, with $x=x(m):=t / m$. From $\dot{x}:=\mathrm{d} x / \mathrm{d} m=-t / m^{2}$ and l'Hôpital's rule, we conclude that

$$
m(\varphi(x)-1)=\frac{\varphi(x)-1}{1 / m} \sim-\frac{\dot{x} \varphi^{\prime}(x)}{1 / m^{2}}=t \varphi^{\prime}(x) \sim t \varphi^{\prime}(0)=\mathrm{i} t \mathrm{E}(X),
$$

where $\varphi^{\prime}(x)=\mathrm{d} \varphi(x) / \mathrm{d} x$. Thus, $\mathrm{E}\left(\mathrm{e}^{\mathrm{i} t Y_{m} / m}\right)$ converges to $\mathrm{e}^{\mathrm{i} t \mathrm{E}(X)}$ as $m$ tends to infinity, for each $t \in \mathbb{R}$. This pointwise convergence of the Fourier transforms is equivalent to the convergence of $Y_{m} / m$, in distribution, to $\mathrm{E}(X)$. As the limiting random variable is constant, this is equivalent to the convergence in probability.

(b) Fix $t \in \mathbb{R}$ and define $\mu_{m}:=m \mathrm{E}(X)$ and $\sigma_{m}^{2}:=m \mathrm{E}\left(X^{2}\right)$ for convenience. Obviously,

$$
\mathrm{E}\left(\mathrm{e}^{\mathrm{i} t\left(Y_{m}-\mu_{m}\right) / \sigma_{m}}\right)=\exp \left(-\frac{\mu_{m}}{\sigma_{m}} \mathrm{i} t+m\left(\varphi\left(\frac{t}{\sigma_{m}}\right)-1\right)\right) .
$$

The Taylor expansion of $\varphi$, together with $\varphi^{\prime}(0)=\mathrm{i} \mathrm{E}(X)$ and $\varphi^{\prime \prime}(0)=-\mathrm{E}\left(X^{2}\right)$, yields

$$
\begin{aligned}
-\frac{\mu_{m}}{\sigma_{m}} \mathrm{i} t+m\left(\varphi\left(\frac{t}{\sigma_{m}}\right)-1\right) \\
=-\frac{\mu_{m}}{\sigma_{m}} \mathrm{i} t+m\left(\varphi^{\prime}(0) \frac{t}{\sigma_{m}}+\frac{\varphi^{\prime \prime}(0)}{2} \frac{t^{2}}{\sigma_{m}^{2}}+o\left(\sigma_{m}^{-2}\right)\right) \\
=\mathrm{i} t \frac{m \mathrm{E}(X)-\mu_{m}}{\sigma_{m}}-\frac{t^{2}}{2} \frac{m \mathrm{E}\left(X^{2}\right)}{\sigma_{m}^{2}}+o(1) \\
=-\frac{1}{2} t^{2}+o(1) .
\end{aligned}
$$

Thus, $\mathrm{E}\left(\mathrm{e}^{\mathrm{i} t\left(Y_{m}-\mu_{m}\right) / \sigma_{m}}\right)$ converges to $\mathrm{e}^{-t^{2} / 2}$ as $m$ tends to infinity. This pointwise convergence of the Fourier transforms implies the convergence in distribution.

If the first moment of $X$ does not exist, then the behavior of $Y_{m}$ for large $m$ can be quite different. For example, if $X$ has Fourier transform $\varphi(t)=\mathrm{e}^{-c|t|}$ (the Cauchy distribution with parameter $c>0$ ), then $\lim _{m \rightarrow \infty} m(\varphi(t / m)-1)=-c|t|$ for all $t \in \mathbb{R}$, i.e. $Y_{m} / m$ converges in distribution to a Cauchy distribution with the same parameter $c$. In particular, in this case $Y_{m} / m$ no longer converges to a constant. There even exist examples in which $Y_{m} / m$ no longer converges at all. The following lemma illustrates this.

Lemma 2.1. If the Fourier transform $\varphi$ of $X$ satisfies $\lim _{t \rightarrow 0}\left|\varphi^{\prime}(t)\right|=\infty$, and if there exists some constant $a \in \mathbb{R}$ such that

$$
\lim _{t \rightarrow 0} t \varphi^{\prime \prime}(t)=-\mathrm{i} a,
$$

then the scaled random variable $Y_{m} /(m \log m)$ converges in probability to a as $m$ tends to infinity, i.e. $\lim _{m \rightarrow \infty} \mathrm{P}\left(\left|Y_{m} /(m \log m)-a\right| \geq \varepsilon\right)=0$ for all $\varepsilon>0$.

Proof. It is sufficient to verify the pointwise convergence of the corresponding Fourier transforms. Thus, for $t \in \mathbb{R}$, consider

$$
\mathrm{E}\left(\mathrm{e}^{\mathrm{i} t Y_{m} /(m \log m)}\right)=\exp (m(\varphi(x)-1)),
$$


with $x \equiv x(m):=t /(m \log m)$. Note that $m$ tends to infinity if and only if $x$ tends to 0 . Moreover,

$$
\dot{x}:=\frac{\mathrm{d} x}{\mathrm{~d} m}=-\frac{t(1+\log m)}{(m \log m)^{2}} \sim-\frac{t}{m^{2} \log m}=-\frac{x}{m} .
$$

L'Hôpital's rule implies that

$$
m(\varphi(x)-1)=-\frac{1-\varphi(x)}{1 / m} \sim \frac{-\dot{x} \varphi^{\prime}(x)}{1 / m^{2}}=-m^{2} \dot{x} \varphi^{\prime}(x) \sim \frac{t \varphi^{\prime}(x)}{\log m} .
$$

Applying l'Hôpital's rule once more yields

$$
m(\varphi(x)-1) \sim \frac{t \dot{x} \varphi^{\prime \prime}(x)}{1 / m} \sim-t x \varphi^{\prime \prime}(x) \sim \mathrm{i} t a,
$$

where, in the last step, condition (2.2) has been used.

If the conditions of Lemma 2.1 are satisfied, then l'Hôpital's rule implies that

$$
\lim _{t \rightarrow 0} \frac{\varphi^{\prime}(t)}{\log |t|}=\lim _{t \rightarrow 0} t \varphi^{\prime \prime}(t)=-\mathrm{i} a .
$$

Therefore, for $t$ close to $0, \varphi^{\prime}(t)$ behaves asymptotically like $-\mathrm{i} a \log |t|$. Under a stronger condition, namely that the function $\varphi^{\prime}(t)+\mathrm{i} a \log |t|$ has certain limits from the left and from the right, the following stronger convergence result holds. Let $\mathcal{B}$ denote the Borel $\sigma$-field on $\mathbb{R}$.

Theorem 2.2. If there exist constants $a \in \mathbb{R}$ and $c>0$ such that

$$
\lim _{t \searrow 0}\left(\varphi^{\prime}(t)+\mathrm{i} a \log |t|\right)=-\mathrm{i} a-c
$$

and

$$
\lim _{t \nearrow 0}\left(\varphi^{\prime}(t)+\mathrm{i} a \log |t|\right)=-\mathrm{i} a+c,
$$

then, as $m$ tends to infinity, the normalized random variable

$$
Z_{m}:=\frac{Y_{m}-a m \log m}{m}=\frac{Y_{m}}{m}-a \log m
$$

converges in distribution to a stable limiting probability measure $\mathrm{Q}$ on $(\mathbb{R}, \mathscr{B})$ uniquely determined via its Fourier transform,

$$
\varphi_{\mathrm{Q}}(t):=\int_{\mathbb{R}} \mathrm{e}^{\mathrm{i} t x} \mathrm{Q}(\mathrm{d} x)=\exp (-c|t|-\mathrm{i} a t \log |t|), \quad t \in \mathbb{R} .
$$

Remark 2.2. The probability measure $\mathrm{Q}$ thus belongs to the well-known class of stable Cauchy laws with parameters $c>0$ and $\beta \in[-1,1]$ (and exponent $\alpha=1$ ) having Fourier transform

$$
\varphi(t)=\exp (-c|t|(1+\mathrm{i} \beta(2 / \pi) \operatorname{sgn}(t) \log |t|)) .
$$

Define $\mu_{m}:=a m \log m$ and $\sigma_{m}:=m$ for convenience. Theorem 2.2 ensures that

$$
\lim _{m \rightarrow \infty} \sup _{x \in \mathbb{R}}\left|\mathrm{P}\left(Y_{m} \leq \sigma_{m} x+\mu_{m}\right)-F(x)\right|=0,
$$


where $F$ denotes the distribution function of Q. Thus, uniformly in $k$, the approximation $\mathrm{P}\left(Y_{m} \leq k\right) \approx F(k / m-\log m)$ holds for large $m$. The probability measure $\mathrm{Q}$ is hence very useful in approximating the distribution of $Y_{m}$.

Obviously, under the conditions of Theorem 2.2, the first moment of $Y_{m}$ does not exist. Hence, the normalizing sequences $\mu_{m}=a m \log m$ and $\sigma_{m}=m$ differ quite dramatically from the 'usual' sequences $\left(\mu_{m}=m \mathrm{E}(X)\right.$ and $\left.\sigma_{m}=\left[m \mathrm{E}\left(X^{2}\right)\right]^{1 / 2}\right)$ known from Theorem 2.1(b). From

$$
\frac{Y_{m}}{m \log m}=\frac{1}{\log m} \frac{Y_{m}-a m \log m}{m}+a,
$$

we conclude, by an application of Slutsky's theorem, that the convergence in Theorem 2.2 is stronger than the convergence $Y_{m} /(m \log m) \rightarrow a$ in probability (see Lemma 2.1).

Proof of Theorem 2.2. Fix $t \in \mathbb{R}$ and define $x:=t / m$ for convenience. Note that

$$
\dot{x}:=\frac{\mathrm{d} x}{\mathrm{~d} m}=-\frac{t}{m^{2}}=-\frac{x}{m} .
$$

The Fourier transform of $Z_{m}$ is

$$
\mathrm{E}\left(\mathrm{e}^{\mathrm{i} t Z_{m}}\right)=\exp (-\mathrm{i} t a \log m+m(\varphi(t / m)-1)) .
$$

Thus, we must verify that

$$
\lim _{m \rightarrow \infty}(-\mathrm{i} t a \log m+m(\varphi(t / m)-1))=-c|t|-\mathrm{i} t a \log |t| .
$$

Multiplying by $1 / t$ shows that this equation is equivalent to

$$
\lim _{m \rightarrow \infty} \frac{\varphi(x)-1-\mathrm{i} a x \log m}{x}=-c \operatorname{sgn}(t)-\mathrm{i} a \log |t| .
$$

L'Hôpital's rule implies that

$$
\begin{aligned}
\frac{\varphi(x)-1-\mathrm{i} a x \log m}{x} & \sim \frac{\dot{x} \varphi^{\prime}(x)-\mathrm{i} a(\dot{x} \log m+x / m)}{\dot{x}} \\
& =\varphi^{\prime}(x)-\mathrm{i} a \log m+\mathrm{i} a \\
& =\varphi^{\prime}(x)+\mathrm{i} a \log |x|-\mathrm{i} a \log |t|+\mathrm{i} a \\
& \sim-c \operatorname{sgn}(t)-\mathrm{i} a \log |t|,
\end{aligned}
$$

by assumption. Thus, as $m$ tends to infinity, the Fourier transform of $Z_{m}$ converges pointwise to the continuous function $t \mapsto \exp (-c|t|-\mathrm{i} a t \log |t|)$. The continuity theorem for Fourier transforms ensures that this function is a Fourier transform of a certain probability measure $\mathrm{Q}$ on $(\mathbb{R}, \mathcal{B})$, and that $Z_{m}$ converges in distribution to $\mathrm{Q}$. Standard results for characteristic functions (for example [10, Theorem 5.7.3]) ensure that $Q$ is a stable distribution with exponent $\alpha=1$

The most simple example is probably the Cauchy distribution $\left(\varphi(t)=\mathrm{e}^{-c|t|}\right)$. In this case, the conditions of Theorem 2.2 are obviously satisfied with $a=0$, and the limiting distribution $\mathrm{Q}$ coincides with the distribution of $X\left(\varphi_{\mathrm{Q}}(t)=\mathrm{e}^{-c|t|}\right)$.

In the following sections, we focus on a special random variable $X$ that satisfies condition (2.2) of Lemma 2.1 and the conditions of Theorem 2.2 with $a=1$ and $c=\frac{1}{2} \pi$. The corresponding compound Poisson distribution is the LD distribution. 


\section{The Luria-Delbrück distribution}

The LD distribution with parameter $m>0$ is the distribution of a special compound-Poissondistributed random variable $Y_{m}$ of the form (2.1), where the random variable $X:=X_{1}$ takes the integer value $k \in \mathbb{N}$ with probability

$$
\alpha_{k}:=\mathrm{P}(X=k)=\frac{1}{k(k+1)}=\frac{1}{k}-\frac{1}{k+1} .
$$

In order to see this, note that $X$ has the generating function

$$
h(s):=\sum_{k=1}^{\infty} \alpha_{k} s^{k}=\sum_{k=1}^{\infty} \frac{s^{k}}{k(k+1)}=1+\frac{(1-s) \log (1-s)}{s} .
$$

From $\mathrm{E}\left(s^{Y_{m}}\right)=\exp (m(h(s)-1))$, it follows that $Y_{m}$ has the generating function (1.1).

The compound Poisson structure of the LD distribution has consequences helpful in applications. For example, it leads to efficient pseudorandom number generators. Let $U$ be uniformly distributed on the open unit interval $(0,1)$. Define $X:=\lfloor 1 / U\rfloor$, where $\lfloor x\rfloor$ denotes the largest integer less than or equal to $x$. Then $X$ has distribution (3.1) and, hence, the following algorithm is applicable. Sample a pseudorandom number $n \in \mathbb{N}_{0}$ from the Poisson distribution with parameter $m$, and then independently sample $n$ pseudorandom numbers $u_{1}, \ldots, u_{n}$ from the uniform distribution on the open unit interval. Then $\left\lfloor 1 / u_{1}\right\rfloor+\cdots+\left\lfloor 1 / u_{n}\right\rfloor$ is a pseudorandom number sampled from the LD distribution with parameter $m$.

For our purposes, it is necessary to study the Fourier transform $\varphi$ of $X$. Obviously,

$$
\begin{aligned}
\varphi(t) & :=\mathrm{E}\left(\mathrm{e}^{\mathrm{i} t X}\right)=\sum_{k=1}^{\infty} \alpha_{k} \mathrm{e}^{\mathrm{i} t k} \\
& =\sum_{k=1}^{\infty} \frac{\mathrm{e}^{\mathrm{i} t k}}{k(k+1)}=\sum_{k=1}^{\infty} \frac{\cos (t k)}{k(k+1)}+\mathrm{i} \sum_{k=1}^{\infty} \frac{\sin (t k)}{k(k+1)}, \quad t \in \mathbb{R} .
\end{aligned}
$$

Using the principal branch of the complex $\log$ arithm $\log z:=\log (|z|)+\mathrm{i} \arg z$, where the $\log$ on the right-hand side of the equation denotes the usual real logarithm and $-\pi<\arg z<\pi$, it follows that

$$
\varphi(t)=1+\frac{\left(1-\mathrm{e}^{\mathrm{i} t}\right) \log \left(1-\mathrm{e}^{\mathrm{i} t}\right)}{\mathrm{e}^{\mathrm{i} t}}
$$

for all $t \in \mathbb{R}$, with the convention $0 \log 0:=0$ for $t=0$. After some algebraic manipulation, the derivatives turn out to be

$$
\varphi^{\prime}(t)=-\mathrm{i}\left(1+\frac{\log \left(1-\mathrm{e}^{\mathrm{i} t}\right)}{\mathrm{e}^{\mathrm{i} t}}\right)
$$

and

$$
\varphi^{\prime \prime}(t)=-\frac{1}{1-\mathrm{e}^{\mathrm{i} t}}-\frac{\log \left(1-\mathrm{e}^{\mathrm{i} t}\right)}{\mathrm{e}^{\mathrm{i} t}}=-\frac{\mathrm{e}^{\mathrm{i} t} /\left(1-\mathrm{e}^{\mathrm{i} t}\right)+\log \left(1-\mathrm{e}^{\mathrm{i} t}\right)}{\mathrm{e}^{\mathrm{i} t}},
$$

where $t \in \mathbb{R} \backslash\{0\}$. Obviously, $\varphi^{\prime}$ and $\varphi^{\prime \prime}$ have singularities at $t=0$. L'Hôpital's rule implies that

$$
\lim _{t \rightarrow 0} \frac{t \mathrm{e}^{\mathrm{i} t}}{1-\mathrm{e}^{\mathrm{i} t}}=\mathrm{i} \quad \text { and } \quad \lim _{t \rightarrow 0} t \log \left(1-\mathrm{e}^{\mathrm{i} t}\right)=0 .
$$

Thus, the second derivative of the Fourier transform of $X$ satisfies the condition $\lim _{t \rightarrow 0} t \varphi^{\prime \prime}(t)=$ $-\mathrm{i}$ and, hence, Lemma 2.1 is applicable with $a=1$. Therefore, $Y_{m} /(m \log m)$ converges in probability to 1 . 


\section{Convergence to the continuous Luria-Delbrück distribution}

An elementary calculation based on (3.2) shows that the conditions of Theorem 2.2 are satisfied with $a=1$ and $c=\frac{1}{2} \pi$. The following result is hence a special case of Theorem 2.2. It can also be found in [2, Equation (15)] and goes back to [13, p. 993].

Theorem 4.1. (Asymptotics of the LD distribution.) As $m$ tends to infinity, the normalized random variable

$$
\frac{Y_{m}-m \log m}{m}=\frac{Y_{m}}{m}-\log m
$$

converges in distribution to a stable limiting probability measure $\mathrm{Q}$ on $(\mathbb{R}, \mathcal{B})$ uniquely determined via its Fourier transform,

$$
\varphi_{\mathrm{Q}}(t):=\int_{\mathbb{R}} \mathrm{e}^{\mathrm{i} t x} \mathrm{Q}(\mathrm{d} x)=(-\mathrm{i} t)^{-\mathrm{i} t}=\exp \left(-\frac{1}{2} \pi|t|-\mathrm{i} t \log |t|\right), \quad t \in \mathbb{R} .
$$

Remark 4.1. The integral calculations of [10, pp. 135-136] show that the Khinchin representation of $\varphi_{\mathrm{Q}}$ has the form

$$
\log \varphi_{\mathrm{Q}}(t)=\mathrm{i} \beta t+\int_{(0, \infty)}\left(\mathrm{e}^{\mathrm{i} t u}-1-\frac{\mathrm{i} t u}{1+u^{2}}\right) \frac{1+u^{2}}{u^{2}} v(\mathrm{~d} u)
$$

where $v$ is Cauchy distributed with density $u \mapsto 1 /\left(1+u^{2}\right)$ and

$$
\beta:=\int_{(0, \infty)}\left(\frac{1}{u\left(1+u^{2}\right)}-\frac{\sin u}{u^{2}}\right) \mathrm{d} u .
$$

Note that $\beta=C-1$, where $C \approx 0.57722$ denotes the Euler constant (see [5, p. 441, Equations 3.783.2 and 3.784.4]). Therefore, the Lévy representation of $\varphi_{\mathrm{Q}}$ is

$$
\log \varphi_{\mathrm{Q}}(t)=\int_{(0, \infty)} \frac{\mathrm{e}^{\mathrm{i} t u}-1-t \sin u}{u^{2}} \lambda(\mathrm{d} u),
$$

i.e. the canonical measure is the Lebesgue measure $\lambda$ restricted to $(0, \infty)$. It is shown in the following corollary that $\mathrm{Q}$ has a density with respect to the Lebesgue measure. Therefore, it is natural to call Q the standard continuous Luria-Delbrück distribution. For completeness, we now present an independent proof of Theorem 4.1 that does not use the derivatives of $\varphi$.

Proof of Theorem 4.1. Fix $t \in \mathbb{R}$ and define $z:=\mathrm{e}^{\mathrm{i} t / m}$ for convenience. The Fourier transform of $Y_{m} / m-\log m$ is

$$
\begin{aligned}
\mathrm{E}\left(\mathrm{e}^{\mathrm{i} t\left(Y_{m} / m-\log m\right)}\right) & =\exp (-\mathrm{i} t \log m+m(\varphi(t / m)-1)) \\
& =\exp \left(-\mathrm{i} t \log m+m \frac{1-z}{z} \log (1-z)\right) .
\end{aligned}
$$

Note that $z=1+O(1 / m)$ and $m(1-z)=-\mathrm{i} t+O(1 / m)$. Thus,

$$
\begin{aligned}
-\mathrm{i} t \log m+m \frac{1-z}{z} \log (1-z) & \sim-\mathrm{i} t \log m-\mathrm{i} t \log (1-z) \\
& \rightarrow-\mathrm{i} t \log (-\mathrm{i} t) .
\end{aligned}
$$


Obviously, $\arg (-\mathrm{i} t)=-\frac{1}{2} \pi \operatorname{sgn} t$ and, hence,

$$
-\mathrm{i} t \log (-\mathrm{i} t)=-\mathrm{i} t(\log |t|+\mathrm{i} \arg (-\mathrm{i} t))=-\mathrm{i} t \log |t|-\frac{1}{2} \pi|t| .
$$

Now proceed as in the proof of Theorem 2.2.

Remark 4.2. (Laplace transform of the standard continuous LD distribution.) An argument similar to that in the previous proof shows that the continuous LD distribution Q has the Laplace transform

$$
\psi_{\mathrm{Q}}(\lambda):=\int_{\mathbb{R}} \mathrm{e}^{-\lambda x} \mathrm{Q}(\mathrm{d} x)=\mathrm{e}^{\lambda \log \lambda}=\lambda^{\lambda}, \quad \lambda \geq 0,
$$

in agreement with the formal substitution of $-\mathrm{i} t$ for $\lambda$ in the Fourier transform (4.1).

Corollary 4.1. (Density of the standard continuous LD distribution.) The continuous LD distribution is absolutely continuous with density

$$
\begin{aligned}
f(x) & =\frac{1}{\pi} \int_{0}^{\infty} \mathrm{e}^{-(\pi / 2) t} \cos (x t+t \log t) \mathrm{d} t \\
& =\frac{1}{\pi} \int_{0}^{\infty} \mathrm{e}^{-x t-t \log t} \sin (\pi t) \mathrm{d} t, \quad x \in \mathbb{R} .
\end{aligned}
$$

In particular, $\sup _{x \in \mathbb{R}} f(x) \leq 2 / \pi^{2} \approx 0.2026$. Moreover, $\lim _{x \rightarrow \infty} x^{2} f(x)=1$.

Proof. Obviously, $\left|\varphi_{\mathrm{Q}}(t)\right|=\exp \left(-\frac{1}{2} \pi|t|\right)$ and, hence, $\int_{\mathbb{R}}\left|\varphi_{\mathrm{Q}}(t)\right| \lambda(\mathrm{d} t)=4 / \pi<\infty$. Therefore, the Fourier inversion theorem ensures that

$$
f(x)=\frac{1}{2 \pi} \int_{\mathbb{R}} \mathrm{e}^{-\mathrm{i} x t} \varphi_{\mathrm{Q}}(t) \lambda(\mathrm{d} t), \quad x \in \mathbb{R},
$$

is a density of Q. Substituting the expression for $\varphi_{\mathrm{Q}}$, splitting the integral into the two integrals over the negative and positive parts of the real axis, and substituting $-t$ for $t$ in the second integral yields

$$
\begin{aligned}
f(x) & =\frac{1}{2 \pi} \int_{0}^{\infty} \mathrm{e}^{-\mathrm{i} x t} \mathrm{e}^{-(\pi / 2) t} \mathrm{e}^{-\mathrm{i} t \log t} \mathrm{~d} t+\frac{1}{2 \pi} \int_{0}^{\infty} \mathrm{e}^{\mathrm{i} x t} \mathrm{e}^{-(\pi / 2) t} \mathrm{e}^{\mathrm{i} t \log t} \mathrm{~d} t \\
& =\frac{1}{\pi} \int_{0}^{\infty} \mathrm{e}^{-(\pi / 2) t} \cos (x t+t \log t) \mathrm{d} t .
\end{aligned}
$$

In particular, $f(x) \leq \pi^{-1} \int_{0}^{\infty} \mathrm{e}^{-(\pi / 2) t} \mathrm{~d} t=2 / \pi^{2}$ for all $x \in \mathbb{R}$. The second integral representation for $f$ follows from standard theory of characteristic functions, for example from Equation (5.8.15) of [10] with $\beta=1$ and $c=\frac{1}{2} \pi$.

To prove that $\lim _{x \rightarrow \infty} x^{2} f(x)=1$, we use classical asymptotics for integrals. One formulation is in [4, Theorem 4.1.6]. Consider the kernel $k:(0, \infty) \rightarrow \mathbb{R}, k(t):=t \mathrm{e}^{-t}$, and its Mellin transform

$$
\check{k}(z):=\int_{(0, \infty)} t^{-z} k(t) \frac{\mathrm{d} t}{t}=\int_{(0, \infty)} t^{-z} \mathrm{e}^{-t} \mathrm{~d} t=\Gamma(1-z),
$$

which converges for at least all $z \in \mathbb{C}$ with $-\infty<\operatorname{Re} z<1$. Furthermore, define the help function $h:(0, \infty) \rightarrow \mathbb{R}$ via

$$
h(t):=\frac{1}{\pi} \exp \left(\frac{\log t}{t}\right) \sin \left(\frac{\pi}{t}\right),
$$


such that the Mellin convolution, $k *_{\mathrm{M}} h$, of $k$ and $h$ satisfies

$$
\begin{aligned}
\left(k *_{\mathrm{M}} h\right)(x) & :=\int_{(0, \infty)} k\left(\frac{x}{u}\right) h(u) \frac{\mathrm{d} u}{u} \\
& =\int_{(0, \infty)} k(x t) h\left(\frac{1}{t}\right) \frac{\mathrm{d} t}{t}=x f(x) \quad \text { for } x>0 .
\end{aligned}
$$

Obviously, $t^{2} h(t)$ is bounded on every interval $(0, a]$ and $h(t) \sim 1 / t$ as $t \rightarrow \infty$. Thus, we can apply Theorem 4.1.6 of [4] (with $\sigma=-2, \tau=0, \rho=-1, l=1, c=1$, and $f=h$ ) to conclude that

$$
x f(x)=\left(k *_{\mathrm{M}} h\right)(x) \sim \frac{\check{k}(-1)}{x}=\frac{\Gamma(2)}{x}=\frac{1}{x} \quad \text { as } x \rightarrow \infty .
$$

Corollary 4.2. (Local asymptotics of the LD distribution.) The discrete LD distribution converges locally to the continuous $L D$ distribution, i.e.

$$
\lim _{m \rightarrow \infty} \sup _{k \in \mathbb{Z}}\left|m \mathrm{P}\left(Y_{m}=k\right)-f\left(\frac{k}{m}-\log m\right)\right|=0 .
$$

Proof. In order to apply Gnedenko's local limit theorem (see, for instance, [4, Theorem 8.4.1]), we have to define the random variables $Y_{m}$ on a common probability space. Let $\left(X_{k}\right)_{k \in \mathbb{N}}$ be a sequence of independent, identically distributed random variables with distribution (3.1) and let $(K(t))_{t \geq 0}$ be a homogeneous Poisson process with unit rate, starting in $K(0)=0$, that is independent of the sequence $\left(X_{k}\right)_{k \in \mathbb{N}}$. Then, for $m \in \mathbb{N}$,

$$
Y_{m}:=\sum_{k=1}^{K(m)} X_{k}
$$

is LD distributed with parameter $m$. Moreover, we can write $Y_{m}=Z_{1}+\cdots+Z_{m}$ in terms of the independent, identically distributed random variables

$$
Z_{i}:=\sum_{k=K(i-1)+1}^{K(i)} X_{k}
$$

i.e. $\left(Y_{m}\right)_{m \in \mathbb{N}}$ is a random walk on the lattice $\mathbb{Z}$ generated by $Z_{1}=Y_{1}$. Theorem 4.1 proves that the distribution function of $Y_{1}$ is in the domain of attraction of the stable law $\mathrm{Q}$. The distribution of $Y_{1}$ is not concentrated on some sublattice of the nonnegative integers, i.e. the maximal span is 1 . Therefore, the conditions of Gnedenko's theorem are satisfied.

Remark 4.3. Theorem 4.1 also ensures that we are able to apply Corollary 8.4.3 of [4]. Therefore, in addition to the above local limit result, we have

$$
\lim _{m \rightarrow \infty} \sum_{k \in \mathbb{Z}}\left|\mathrm{P}\left(Y_{m}=k\right)-\frac{1}{m} f\left(\frac{k}{m}-\log m\right)\right|=0 .
$$

In Corollary 4.1, we have found integral representations for the density $f$. Computing the corresponding distribution function $F$ would hence require us to integrate twice. The following proposition presents a more useful representation for $F$, avoiding double integration. For further useful integral representations we refer the reader to [15, p. 78, Remark 1]. 
Proposition 4.1. (Distribution function of the continuous LD distribution.) The distribution function $F$ of the continuous LD distribution satisfies

$$
F(a)=\frac{1}{2}+\frac{1}{\pi} \int_{0}^{\infty} \frac{\mathrm{e}^{-(\pi / 2) t}}{t} \sin (a t+t \log t) \mathrm{d} t, \quad a \in \mathbb{R} .
$$

Moreover, $\lim _{x \rightarrow \infty} x(1-F(x))=1$.

Proof. Let $a, b \in \mathbb{R}$ with $a \leq b$. Integrating (4.2) from $a$ to $b$ and interchanging the integrals (using Fubini's theorem) leads to

$$
\begin{aligned}
F(b)-F(a) & =\frac{1}{\pi} \int_{0}^{\infty} \mathrm{e}^{-(\pi / 2) t} \int_{a}^{b} \cos (x t+t \log t) \mathrm{d} x \mathrm{~d} t \\
& =\frac{1}{\pi} \int_{0}^{\infty} \frac{\mathrm{e}^{-(\pi / 2) t}}{t} \sin (b t+t \log t) \mathrm{d} t-\frac{1}{\pi} \int_{0}^{\infty} \frac{\mathrm{e}^{-(\pi / 2) t}}{t} \sin (a t+t \log t) \mathrm{d} t
\end{aligned}
$$

Thus, as $\lim _{b \rightarrow \infty} F(b)=1$, the proposition is established if we can verify that

$$
\lim _{b \rightarrow \infty} \int_{0}^{\infty} \frac{\mathrm{e}^{-(\pi / 2) t}}{t} \sin (b t+t \log t) \mathrm{d} t=\frac{\pi}{2} .
$$

As $\sin (x+y)=\sin x \cos y+\cos x \sin y$, it suffices to prove that

$$
\lim _{b \rightarrow \infty} \int_{0}^{\infty} \frac{\mathrm{e}^{-(\pi / 2) t}}{t} \sin (b t) \cos (t \log t) \mathrm{d} t=\frac{\pi}{2}
$$

and that

$$
\lim _{b \rightarrow \infty} \int_{0}^{\infty} \frac{\mathrm{e}^{-(\pi / 2) t}}{t} \cos (b t) \sin (t \log t) \mathrm{d} t=0 .
$$

The integral on the left-hand side of (4.4) is the Mellin convolution

$$
\left(k *_{\mathrm{M}} h\right)(b):=\int_{(0, \infty)} k(b t) h\left(\frac{1}{t}\right) \frac{\mathrm{d} t}{t}
$$

of the kernel $k(t):=\sin (t)$ and the function

$$
h(t):=\exp \left(-\frac{\pi}{2 t}\right) \cos \left(\frac{\log (1 / t)}{t}\right), \quad t>0 .
$$

Obviously, $|h(t)| \leq 1$ and $\lim _{t \rightarrow \infty} h(t) \rightarrow 1$. It is well known (see, for instance, [4, p. 206, Equation (4.3.1a)]) that the Mellin transform

$$
\check{k}(z):=\int_{(0, \infty)} t^{-z} k(t) \frac{\mathrm{d} t}{t}=\int_{(0, \infty)} t^{-z-1} \sin (t) \mathrm{d} t
$$

of $k$ converges for at least all $z \in \mathbb{C}$ with $|\operatorname{Re}(z)|<1$. Thus, we can apply Theorem 4.1.6 of [4] (with $\sigma=-\frac{1}{2}, \tau=\frac{1}{2}, \rho=0, l=1, c=1$, and $f=h$ ) to conclude that $\left(k *_{\mathrm{M}} h\right)(b)$ converges to $\breve{k}(0)=\int_{(0, \infty)} t^{-1} \sin (t) \mathrm{d} t=\frac{1}{2} \pi$ as $b$ tends to infinity. Thus, we have verified (4.4).

To prove (4.5), consider the kernel $k(t):=\cos (t)$. Its Mellin transform,

$$
\check{k}(z)=\int_{(0, \infty)} t^{-z-1} \cos (t) \mathrm{d} t,
$$


converges at least in the strip $-1<\operatorname{Re}(z)<0$. Thus, Theorem 4.1.6 of [4], applied to the function

$$
h(t):=\exp \left(-\frac{\pi}{2 t}\right) \sin \left(\frac{\log (1 / t)}{t}\right), \quad t>0
$$

(with $\sigma=-\frac{3}{4}, \tau=-\frac{1}{4}, \rho=-\frac{1}{2}, l(x)=\log (x)$, and $c=1$ ), yields

$$
\left(k *_{\mathrm{M}} h\right)(b) \sim \breve{k}\left(-\frac{1}{2}\right) b^{-1 / 2} \log (b) \rightarrow 0 \quad \text { as } b \rightarrow \infty,
$$

and (4.5) follows.

L'Hôpital's rule implies that

$$
\lim _{x \rightarrow \infty} x(1-F(x))=\lim _{x \rightarrow \infty} x^{2} f(x)=1,
$$

by Corollary 4.1 .

Definition 4.1. Let $a \in \mathbb{R}$ and $b>0$ and let $F$ denote the distribution function of the standard continuous LD distribution. The probability measure that corresponds to the linear transformed distribution function $F_{a, b}(x):=F((x-a) / b)$ is called the continuous $L D$ distribution with parameters $a$ and $b$. We denote this probability measure by $\mathrm{Q}_{a, b}$.

Remark 4.4. From Theorem 4.1, it follows that the probability measure $\mathrm{Q}_{a, b}$ has the Fourier transform

$$
\varphi_{a, b}(t)=\mathrm{e}^{\mathrm{i} t a} \varphi_{0,1}(b t)=\exp \left(\mathrm{i} t a-\frac{1}{2} \pi b|t|-\mathrm{i} b t \log (b|t|)\right) .
$$

As the standard continuous LD distribution is stable, it follows that, for all parameters $a_{1}, a_{2} \in \mathbb{R}$ and $b_{1}, b_{2}>0$, there exist two other parameters $a \in \mathbb{R}$ and $b>0$ such that the convolution property

$$
\mathrm{Q}_{a_{1}, b_{1}} \star \mathrm{Q}_{a_{2}, b_{2}}=\mathrm{Q}_{a, b}
$$

holds. The following corollary determines $a$ and $b$ explicitly.

Corollary 4.3. Let $a_{1}, a_{2} \in \mathbb{R}$ and $b_{1}, b_{2}>0$. The continuous LD distribution satisfies the convolution property (4.6) with $b=b_{1}+b_{2}$ and

$$
a=a_{1}+a_{2}+b \log b-b_{1} \log b_{1}-b_{2} \log b_{2} .
$$

Proof. This follows from the equivalent equation $\varphi_{a_{1}, b_{1}}(t) \varphi_{a_{2}, b_{2}}(t)=\varphi_{a, b}(t), t \in \mathbb{R}$, for the corresponding Fourier transforms, which can be verified easily.

\section{Final remarks and discussion}

The density $f$ of the continuous LD distribution is well known from the literature. Kepler and Oprea [7, Equation (32)] discovered $f$ using a different approach, starting with a class of discrete LD distributions with characteristic functions originally derived by Bartlett [3]. Both integral representations for $f((4.2)$ and (4.3)) can be integrated numerically. We prefer to use (4.2), as the integrand $\mathrm{e}^{-(\pi / 2) t} \cos (x t+t \log t)$ is bounded by the decreasing function $t \mapsto \mathrm{e}^{-(\pi / 2) t}$ no matter how $x$ is chosen. Applying [10, Equation (5.8.16)] shows that $f$ has the Taylor expansion $f(x)=(1 / \pi) \sum_{k=0}^{\infty} a_{k} x^{k}, x \in \mathbb{R}$, with coefficients

$$
a_{k}:=\frac{(-1)^{k}}{k !} \int_{0}^{\infty} t^{k} \sin (\pi t) \mathrm{e}^{-t \log t} \mathrm{~d} t .
$$


The new results of the present paper are the convergence results for compound Poisson distributions presented in Section 2. Theorem 2.2, in particular, presents the normalizing sequences $\mu_{m}=a m \log m$ and $\sigma_{m}=m$. We have applied these results to the discrete LD distribution (Theorem 4.1), which leads to integral representations for the density and the distribution function of the continuous LD distribution. Moreover, local convergence holds (Corollary 4.2).

The convergence results also differ from the limiting results of Angerer [1]. He considered versions of the standard LD distribution having finite moments, which are more appropriate for biological applications but which lead to discrete limiting distributions.

The density $f$, together with Theorem 4.1 , is quite useful in deriving approximative results for the discrete LD distribution with parameter $m$. For example, the median and the mode are approximately given by $\left[m\left(\log m+x_{\text {median }}\right)\right]$ and $\left[m\left(\log m+x_{\text {mode }}\right)\right]$, respectively, where $x_{\text {median }}$ and $x_{\text {mode }}$ denote the median and the mode of $f$. Note that the mode of $f$ is uniquely determined, as all stable distributions are unimodal (by Theorem 5.10.1 of [10], due to Ibragimov and Czernin). Numerical analysis shows that $x_{\text {median }} \approx 1.35$ and $x_{\text {mode }} \approx-0.23$. These values reflect the skewness of the continuous LD distribution.

Theorem 4.1 and Corollary 4.2 might also be helpful in estimating the parameter $m$ of the discrete LD distribution and in providing approximative confidence intervals for $m$, but we do not want to go into detail here. In this context, we also refer the reader to [7] and [8].

\section{Acknowledgements}

The author is grateful to the Erwin Schrödinger International Institute for Mathematical Physics (ESI) in Vienna for support during a stay in winter 2002-2003, where part of this work was done. Furthermore, the author thanks an anonymous referee for helpful comments on local convergence and classical integral asymptotics.

\section{References}

[1] Angerer, W. P. (2001). An explicit representation of the Luria-Delbrück distribution. J. Math. Biol. 42, $145-174$.

[2] Angerer, W. P. (2001). A note on the evaluation of fluctuation experiments. Mutation Res. 479, $207-224$.

[3] Bartlett, M. S. (1978). An Introduction to Stochastic Processes, 3rd edn. Cambridge University Press.

[4] Bingham, N. H., Goldie, C. M. And Teugels, J. L. (1987). Regular Variation. Cambridge University Press.

[5] Gradshteyn, I. S. And Ryzhik, I. M. (2000). Tables of Integrals, Series, and Products, 6th edn. Academic Press, San Diego, CA.

[6] Kemp, A. W. (1994). Comments on the Luria-Delbrück distribution. J. Appl. Prob. 31, 822-828.

[7] Kepler, T. B. AND Oprea, M. (2001). Improved inference of mutation rates. I. An integral representation for the Luria-Delbrück distribution. Theoret. Pop. Biol. 59, 41-48.

[8] Kepler, T. B. ANd Oprea, M. (2001). Improved inference of mutation rates. II. Generalization of the LuriaDelbrück distribution. Theoret. Pop. Biol. 59, 49-59.

[9] LEA, D. E. AND Coulson, C. A. (1949). The distribution of the number of mutants in bacterial populations. $J$. Genet. 49, 264-285.

[10] LuKacs, E. (1970). Characteristic Functions, 2nd edn. Griffin, London.

[11] LuRIA, S. E. AND DELBRÜCK, M. (1943). Mutations of bacteria from virus sensitivity to virus resistance. Genetics 28, 491-511.

[12] Ma, W. T., SAndri, G. vH. and SARKar, S. (1992). Analysis of the Luria-Delbrück distribution using discrete convolution powers. J. Appl. Prob. 29, 255-267.

[13] Pakes, A. G. (1993). Remarks on the Luria-Delbrück distribution. J. Appl. Prob. 30, 991-994.

[14] Prodinger, H. (1996). Asymptotics of the Luria-Delbrück distribution via singularity analysis. J. Appl. Prob. 33, 282-283.

[15] Zolotarev, V. M. (1986). One-Dimensional Stable Distributions. American Mathematical Society, Providence, RI. 